\title{
The effects of volume of interest delineation on MRI-based radiomics analysis: evaluation with two disease groups
}

Xiao Zhang ${ }^{1}$, Liming Zhong ${ }^{1}$, Bin Zhang ${ }^{2}$, Lu Zhang ${ }^{3}$, Haiyan Du' ${ }^{1}$, Lijun Lu' ${ }^{1}$ Shuixing Zhang ${ }^{2^{*}}$, Wei Yang ${ }^{1^{*}}$ (D) and Qianjin Feng ${ }^{1}$

\begin{abstract}
Background: Manual delineation of volume of interest (VOI) is widely used in current radiomics analysis, suffering from high variability. The tolerance of delineation differences and possible influence on each step of radiomics analysis are not clear, requiring quantitative assessment. The purpose of our study was to investigate the effects of delineation of VOls on radiomics analysis for the preoperative prediction of metastasis in nasopharyngeal carcinoma (NPC) and sentinel lymph node (SLN) metastasis in breast cancer.

Methods: This study retrospectively enrolled two datasets (NPC group: 238 cases; SLN group: 146 cases). Three operations, namely, erosion, smoothing, and dilation, were implemented on the VOls accurately delineated by radiologists to generate diverse VOI variations. Then, we extracted 2068 radiomics features and evaluated the effects of VOI differences on feature values by the intra-class correlation coefficient (ICC). Feature selection was conducted by Maximum Relevance Minimum Redundancy combined with $0.632+$ bootstrap algorithms. The prediction performance of radiomics models with random forest classifier were tested on an independent validation cohort by the area under the receive operating characteristic curve (AUC).

Results: The larger the VOls changed, the fewer features with high ICCS. Under any variation, SLN group showed fewer features with ICC $\geq 0.9$ compared with NPC group. Not more than 15\% top-predictive features identical to the accurate VOIs were observed across feature selection. The differences of AUCs of models derived from VOls across smoothing or dilation with 3 pixels were not statistically significant compared with the accurate VOls $(p>$ 0.05 ) except for T2-weighted fat suppression images (smoothing: 0.845 vs. $0.725, p=0.001$; dilation: 0.800 vs. 0.725 , $p=0.042$ ). Dilation with 5 and 7 pixels contributed to remarkable AUCS in SLN group but the opposite in NPC group. The radiomics models did not perform well when tested by data from other delineations.
\end{abstract}

Conclusions: Differences in delineation of VOls affected radiomics analysis, related to specific disease and MRI sequences. Differences from smooth delineation or expansion with 3 pixels width around the tumors or lesions were acceptable. The delineation for radiomics analysis should follow a predefined and unified standard.

Keywords: Radiomics, Magnetic resonance imaging, Breast cancer, Nasopharyngeal carcinoma, Preoperative prediction, Segmentation

\footnotetext{
*Correspondence: shui7515@126.com; weiyanggm@gmail.com

${ }^{2}$ Department of Radiology, The First Affiliated Hospital, Jinan University, No.

613 Huangpu West Road, Tianhe District, Guangzhou 510627, Guangdong,

China

${ }^{1}$ School of Biomedical Engineering, Southern Medical University, No.1023

Shatai Road, Baiyun District, Guangzhou 510515, Guangdong, China

Full list of author information is available at the end of the article
}

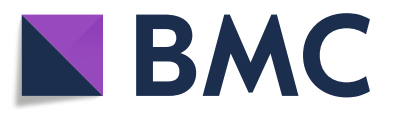

(c) The Author(s). 2019 Open Access This article is distributed under the terms of the Creative Commons Attribution 4.0 International License (http://creativecommons.org/licenses/by/4.0/), which permits unrestricted use, distribution, and

reproduction in any medium, provided you give appropriate credit to the original author(s) and the source, provide a link to the Creative Commons license, and indicate if changes were made. The Creative Commons Public Domain Dedication waiver (http://creativecommons.org/publicdomain/zero/1.0/) applies to the data made available in this article, unless otherwise stated. 


\section{Background}

As an emerging non-invasive tool, radiomics has shown gratifying performance in phenotype diagnosis and classification [1, 2], tumor prognosis [3, 4], treatment decision $[5,6]$, and molecular marker estimation $[7,8]$ by permitting comprehensive quantification tumor heterogeneity on radiographic imaging [9-11]. The process mainly consists of six consecutive steps including image acquisition, image preprocessing, tumor segmentation, feature extraction, feature selection, and radiomics model development. Each step can be an uncertain factor contributing to an unreasonable result due to a lack of standardization in radiomics analysis. Recent studies have focused on identifying the factors that affect radiomics analysis. Vallieres et al. investigated the impact of six parameters of feature extraction on the prediction of lung metastases in soft-tissue sarcomas of the extremities [12]. Lu et al. evaluated the effects of segmentation and discretization methods on radiomics features in 2-deoxy-2-[18F] fluoro-D-glucose and [11C] methyl-choline positron emission tomography/computed tomography (PET/CT) imaging of nasopharyngeal carcinoma (NPC) [13]. In the process of image preprocessing for patients with head and neck cancers, BagherEbadian et al. evaluated changes in radiomics features from images subject to smoothing, sharpening, and noise relative to baseline datasets [14]. In a recent study, Shiri et al. considered the need of reliable feature values against image reconstruction and assessed the variability of radiomics features extracted from multi-scanner phantom and patient $\mathrm{PET} / \mathrm{CT}$ images over a wide range of different reconstruction settings [15].

Among the factors that affect radiomics analysis, delineation of tumors or lesions occupy an important position, as the volume of interest (VOI) is directly used to extract quantitative features [9]. The accuracy may affect subsequent radiomics analysis. Usually, VOIs are manually outlined by radiologists with labor intensive as well as time-consuming. The work in [16] showed that the delineation of VOIs for radiotherapy currently was imprecise with high inter-operator variability, even for experienced observers. Most prior studies have focused solely on the effects of inter-observer variability in manual tumor delineation to identify radiomics features with high robustness $[17,18]$. In fact, quantification of tumor delineation and tolerance assessment of the differences are likely more important in developing standardized research. Recently, Kocal et al. [19] determined the influence of segmentation with margin shrinkage of $2 \mathrm{~mm}$ on CT-based radiomics analysis for distinguishing low and high nuclear grade renal clear cell carcinomas (RcCCs). However, in most cases, delineation tends to overestimate the lesion volume to ensure that the entire lesion is identified [20]. The delineation differences that can be accepted and possible influence on radiomics analysis have not been unexplored, requiring quantitative assessment.

The aim of this work is to investigate the effects of delineation of VOIs on each step of radiomics analysis in detail, including feature extraction, feature selection, and prediction performance of radiomics models. Simultaneously, the tolerance of delineation differences of VOIs was assessed for reference in radiomics analysis.

\section{Methods \\ Patients}

Two datasets were collected to investigate the effects of delineation of VOIs on radiomics analysis. The first problem is to distinguish whether metastasis occurs in patients with NPC before radiotherapy. In clinical practice, the majority of NPC patients with metastasis before radiotherapy suffer from poor prognosis [21-23]. Hence, it will be beneficial to improve prognosis if the risk of transfer before treatment can be distinguished accurately and take timely intervention on patients with high risk of metastasis. Our study retrospectively recruited 238 patients with NPC who had been diagnosed by histopathology between August 2009 and January 2013. All patients were divided into two groups in accordance with the metastasis status: (i) metastasizing (TM) group with 126 patients; (ii) non-metastasizing (NM) group with 112 patients.

The second problem is the prediction of sentinel lymph node (SLN) metastasis in patients with breast cancer, as described in [24]. It is of great significance using radiomics analysis to predict SLN metastasis for treatment decision making in breast cancer. A total of 146 consecutive patients with histologically confirmed breast cancer between March 2014 and June 2016 were retrospectively enrolled in this work. The patients consisted of two groups on the basis of SLN metastasis: (i) TM group with 55 patients; (ii) NM group with 91 patients. The inclusion criteria are available in Additional file 1: Note S1.

The patients were divided into a strictly training cohort for radiomics model building and an independent validation cohort (25\% in NPC group and $33 \%$ in SLN group) for evaluating the final prediction performance. The detailed demographic characteristics and clinical information are summarized in Table 1.

\section{Image acquisition protocol}

All analyses were carried out in accordance with the relevant guidelines and regulations, and the requirement to obtain informed consent was waived. This retrospective study was approved by the local institutional review board. 
Table 1 Demographic characteristics and clinical information of two disease groups

\begin{tabular}{|c|c|c|c|c|c|c|c|}
\hline \multirow{2}{*}{$\frac{\text { Dataset }}{\text { NPC group }}$} & \multirow[t]{2}{*}{ Characteristic } & \multicolumn{3}{|c|}{ Training cohort } & \multicolumn{3}{|c|}{ Independent validation cohort } \\
\hline & & $\mathrm{NM}(n=84)$ & $\mathrm{TM}(n=95)$ & $p$-Value & $\mathrm{NM}(n=28)$ & $\mathrm{TM}(n=31)$ & $p$-Value \\
\hline & Sex & & & 0.903 & & & 0.398 \\
\hline & Male & 63 & 72 & & 21 & 26 & \\
\hline & Female & 21 & 23 & & 7 & 5 & \\
\hline & Mean age (SD) & $43.95(11.72)$ & $45.40(10.26)$ & 0.394 & $40.54(9.74)$ & $45.10(10.27)$ & 0.121 \\
\hline & Histologic grade & & & $<0.001^{*}$ & & & $0.009^{*}$ \\
\hline & I & $3(3.57 \%)$ & $0(0.0 \%)$ & & $1(3.57 \%)$ & $0(0.0 \%)$ & \\
\hline & $\|$ & $9(10.72 \%)$ & $4(4.21 \%)$ & & $6(21.43 \%)$ & $2(6.45 \%)$ & \\
\hline & III & $47(55.95 \%)$ & $19(20.0 \%)$ & & $14(50.0 \%)$ & $8(25.81 \%)$ & \\
\hline & IV & $25(29.76 \%)$ & $72(75.79 \%)$ & & $7(25.0 \%)$ & $21(67.74 \%)$ & \\
\hline & Metastatic sites & & & - & & & - \\
\hline & Lung & - & $36(33.03 \%)$ & & - & $11(30.56 \%)$ & \\
\hline & Liver & - & $30(27.52 \%)$ & & - & $12(33.33 \%)$ & \\
\hline & Bone & - & $43(39.45 \%)$ & & - & $13(36.11 \%)$ & \\
\hline \multirow[t]{11}{*}{ SLN group } & & $\mathrm{NM}(n=60)$ & TM $(n=37)$ & $p$-Value & $N M(n=31)$ & TM $(n=18)$ & $p$-Value \\
\hline & Mean age (SD) & $46.70(11.85)$ & 46.59 (11.04) & 0.935 & 47.32 (9.18) & $50.33(10.06)$ & 0.339 \\
\hline & Histologic grade & & & 0.261 & & & 0.254 \\
\hline & I & $7(11.7 \%)$ & $2(5.4 \%)$ & & $7(22.6 \%)$ & $1(5.6 \%)$ & \\
\hline & $\|$ & 23 (38.3\%) & 20 (54.1\%) & & 12 (38.7\%) & 7 (38.9\%) & \\
\hline & III & $30(50.0 \%)$ & 15 (40.5\%) & & $12(38.7 \%)$ & $10(55.6 \%)$ & \\
\hline & HER2 & & & 0.592 & & & 0.061 \\
\hline & Positive & $21(35.0 \%)$ & $11(29.7 \%)$ & & $6(19.4 \%)$ & $8(44.4 \%)$ & \\
\hline & Negative & 39 (65.0\%) & $26(70.3 \%)$ & & 25 (80.6\%) & $10(55.6 \%)$ & \\
\hline & Ki67 (SD) & 36.30 (24.34) & $26.32(15.61)$ & 0.100 & 30.68 (25.19) & $35.06(27.26)$ & 0.545 \\
\hline & ADC (SD) & $0.86(0.20)$ & $0.82(0.16)$ & 0.331 & $0.84(0.16)$ & $0.86(0.19)$ & 0.442 \\
\hline
\end{tabular}

TM metastasizing, NM non-metastasizing, HER2 human epidermal growth factor receptor 2, Ki67 proliferation index, ADC apparent diffusion coefficient

${ }^{*} p<0.05$ representing statistically significant difference in NM and TM group

a) NPC group: All patients had scanned axial contrastenhanced T1-weighted (CET1-w) and T2-weighted (T2-w) images acquired from a 1.5-T GE scanner (Signa EXCITE HD, TwinSpeed, GE Healthcare, Milwaukee, WI, USA) and a 1.5-T Philips scanner (Achieva, Philips Healthcare, The Netherlands). The GE MRI acquisition parameters were as follows: CET1- $w$ images (TR/TE: 410/Min Full ms, FOV = $230 \times 230 \mathrm{~mm}^{2}, \mathrm{NEX}=2.0$, slice thickness $=4 \mathrm{~mm}$, spacing $=1 \mathrm{~mm}$ ); T2-w images (TR/TE: $5000 / 85 \mathrm{~ms}$, $\mathrm{FOV}=230 \times 230 \mathrm{~mm}^{2}, \mathrm{NEX}=2.0$, slice thickness $=$ $4 \mathrm{~mm}$, spacing $=1 \mathrm{~mm}$ ). The Philips MRI acquisition parameters were as follows: CET1-w images (TR/TE: $636 / 20 \mathrm{~ms}, \mathrm{FOV}=220 \times 220 \mathrm{~mm}^{2}$, NEX $=$ 4.0, slice thickness $=4.5 \mathrm{~mm}$, spacing $=1 \mathrm{~mm}$ ); $\mathrm{T} 2-\mathrm{w}$ images (TR/TE: $3700 / 100 \mathrm{~ms}$, FOV $=220 \times 220$ $\mathrm{mm}^{2}, \mathrm{NEX}=3.0$, slice thickness $=5 \mathrm{~mm}$, spacing $=$ $1 \mathrm{~mm})$.

b) SLN group: All patients underwent pretreatment T2-weighted fat suppression (T2-FS) and diffusion- weighted images (DWI) scan. The anatomical MRI data were acquired on a 1.5 - $\mathrm{T}$ MR scanner (Achieva, Philips Healthcare, Best, The Netherlands) equipped with a 4-channel SENSE breast coil in prone position. Axial DWI with bilateral breast coverage were obtained $(\mathrm{TR} / \mathrm{TE}=5065 /$ $66 \mathrm{~ms}, \mathrm{FOV}=300 \times 300 \mathrm{~mm}^{2}$, matrix $=200 \times 196$, slice thickness $=5 \mathrm{~mm}$, slice gap $=1 \mathrm{~mm}$, $\mathrm{b}$ values of 0 and $1000 \mathrm{~s} / \mathrm{mm}^{2}$ ) by using single-shot spin-echo echo-planar imaging. T2-FS images of breast were collected $(\mathrm{TR} / \mathrm{TE}=3400 / 90 \mathrm{~ms}, \mathrm{FOV}=320 \times 260$ $\mathrm{mm}^{2}$, matrix $=348 \times 299$, slice thickness $=3 \mathrm{~mm}$, slice gap $=0.3 \mathrm{~mm}$ ).

\section{Image pre-processing}

As for the subjects in two datasets enrolled in our study, multi-sequence MR images are required from several MR scanners with different protocols, hence image standardization are essential for all images to avoid the inhomogeneity. Prior to analyzing MR images, additional 
image standardization involving bias field correction and intensity normalization were conducted to avoid inhomogeneity. First, the N4ITK algorithm [25] was applied to remove the bias field artifacts in the MR images. Subsequently, intensity normalization [26] was utilized to reduce the variability across image acquisitions from different manufactures. Fig. 1 illustrates the schematic framework of the radiomics analysis in this work.

\section{Volume of interest segmentation}

All MR images were imported into the ITK-SNAP software designed by Yushkevich et al. [27] to define the VOI of each tumor. The tumor contours were individually first outlined slice-by-slice by two radiologists (Z.L., 4 years of experience, and Z.B., 6 years of experience) and then reviewed by a senior radiologist (Z.S., 12 years of experience). Any disagreement between the readers was discussed until a final consensus was generated. During the session 30 cases randomly selected from each dataset were used for the inter-observer analysis of the segmentation. For each selected region of interest (ROI), the smallest rectangle that best fits the tumor region was used to calculate margin distance of two kinds of manual segmentation in four directions (up, down, left, and right), resulting in multiple calculated values (number of selected ROIs $\times 4$ ) for analysis together.

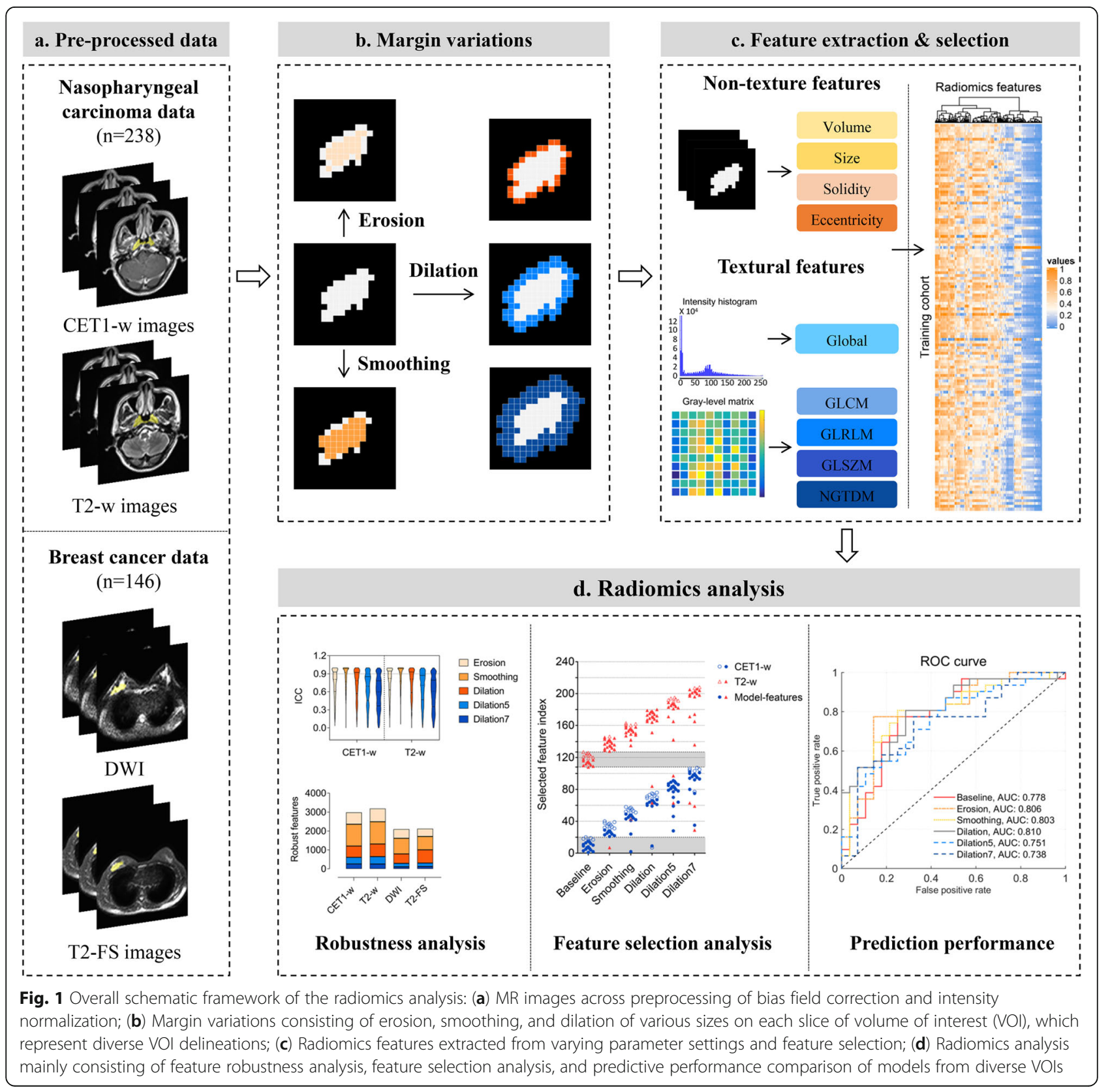




\section{Changes of volume of interest}

On the basis of the original segmented regions, erosion, dilation, and smoothing were performed on the VOIs slice-by-slice to generate diverse VOIs. For the dilation operation, the radius sizes (number of pixels) of the circular structural element to dilate the VOIs were separately set as 3, 5, and 7. Given that certain tumors were extremely small, the size for the erosion operation was only set to 3. Image smoothing for VOIs was implemented by a Gaussian smoothing filter configured with correlation operator, where sigma was set as 3 and the template size was $7 \times 7$. Pixel values outside the bounds of the region of interest were set to the value of the nearest border. The three types of operations were respectively implemented using the functions imdilate, imerode, and imfilter of MATLAB version 8.5 (MathWorks, R2015a). No additional processing was implemented on the contours. For the sake of analysis, five operations on VOIs were abbreviated as Erosion, Smoothing, Dilation, Dilation5, Dilation7, respectively. The VOIs accurately delineated by radiologists were denoted as Baseline. Fig. 2 exemplifies the VOI in a single slice of the original tumor and presents the corresponding drawing of partial enlargement under different operations simultaneously. The degree of tumor volume change of diverse delineations in relation to the accurate delineation is summarized in Table 2.

\section{Feature extraction}

A total of 2068 radiomics features were extracted for each VOI. In reference to [12], four non-texture features that describe the geometric characteristics were calculated, including tumor volume, size, solidity, and eccentricity. In view of the effects of varying extraction parameters on texture features, three extraction parameters, respectively, isotropic voxel size, quantization of gray levels, and quantization algorithm, were adopted, thereby leading to 2064 textural features for each patient. The textural features consisted of Global (extracted from the intensity histogram with 100 bins of the tumor region), grey-level co-occurrence matrix (GLCM), grey-level run length matrix (GLRLM), grey-level size zone matrix (GLSZM) and neighbourhood grey-tone difference matrix (NGTDM) [28-30]. The extraction was conducted with a MATLAB toolkit for radiomics analysis (https://github.com/mvallieres/radiomics). The detailed extraction parameters and description are available in Additional file 1: Note S2 and Table S1.

\section{Feature selection}

The feature selection was performed within the training cohort. Maximum Relevance Minimum Redundancy (mRMR) [31], which has good trade-off between the maximum relevance and minimum redundancy, was firstly explored to identify a well-ranked feature set that included 100 features. Referring to [12, 32], the $0.632+$ bootstrap method combined with the area under the receiver operating characteristic curve (AUC) metric were adopted to evaluated the predictability of features (Additional file 1: Note S3). One thousand iterations were performed with $63.2 \%$ random data resampling

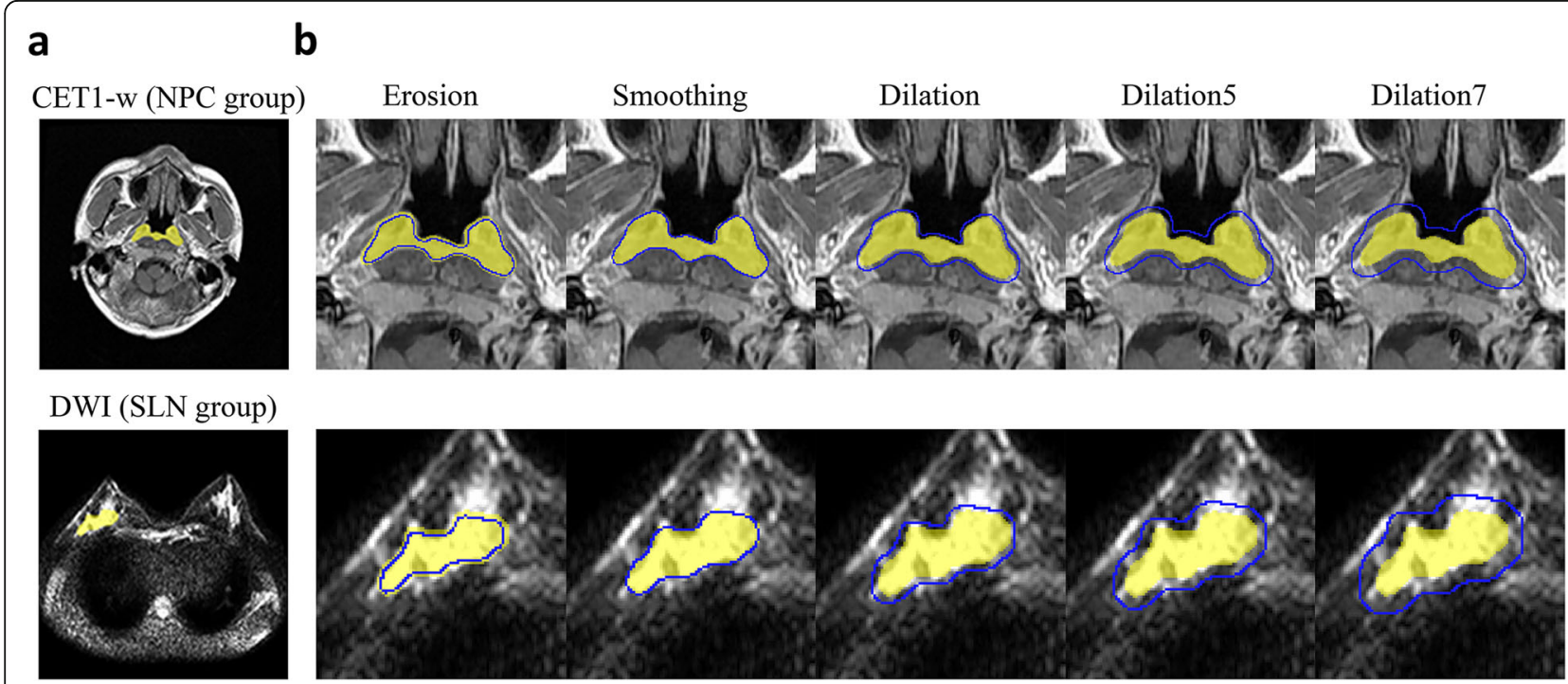

Fig. 2 Segmentation of volume of interest and differences in processing: (a) Example illustrating the VOI in a single slice of tumor delineated by the radiologists (indicated in yellow); (b) Corresponding drawing of partial enlargement under diverse operations (indicated in blue). Erosion represents erosion operation on VOls while Smoothing represents smoothing operation. Dilation, Dilation5, and Dilation7 indicate dilation operation, for which the size of structural elements is set as 3, 5, and 7, respectively. Application of various operations to VOls slice-by-slice corresponds to diverse delineations 
Table 2 Tumor volume change under diverse operations in relation to the accurately outlined tumor

\begin{tabular}{lllllll}
\hline Dataset & & Erosion & Smoothing & Dilation & Dilation5 & Dilation7 \\
\hline NPC group & CET1-W & $0.767(0.757-0.777)$ & $0.991(0.989-0.992)$ & $1.350(1.334-1.366)$ & $1.606(1.579-1.634)$ & $1.976(1.932-2.021)$ \\
& T2-W & $0.745(0.734-0.757)$ & $0.987(0.985-0.988)$ & $1.388(1.369-1.407)$ & $1.675(1.641-1.708)$ & $2.089(2.035-2.144)$ \\
SLN group & DWI & $0.664(0.639-0.674)$ & $0.959(0.952-0.964)$ & $1.578(1.531-1.598)$ & $2.080(1.979-2.107)$ & $2.858(2.654-2.882)$ \\
& T2-FS & $0.788(0.773-0.802)$ & $0.985(0.983-0.987)$ & $1.333(1.307-1.358)$ & $1.609(1.560-1.658)$ & $2.019(1.933-2.105)$ \\
\hline
\end{tabular}

Note that the value (mean with $95 \%$ confidence interval) in the table represents the ratio of the tumor volume after corresponding operations to the original volume

Erosion erosion operation, Smoothing smoothing operation, Dilation dilation with structural element radius size of 3, Dilation5 dilation with structural element radius size of 5, Dilation7 dilation with structural element radius size of 7

from the training cohort between runs. The features selected in the previous step were ranked through maximizing the $0.632+$ bootstrap AUC to determine the final twenty top-predictive features that maximally distinguished two classes.

\section{Development of radiomics model}

Once the discriminative features were identified, radiomics models were built based on different feature sets. A new feature set was composed when one feature from higher to lower rank was added, which contributed to 20 radiomics models. We used the random forest classifier [33] to evaluate the capability of foregoing radiomics models across 10 -fold cross-validation in the training cohort, with 150 decision trees used for training ultimately. The model that possessed the most superior properties was determined for further analysis.

\section{Statistical analysis}

First, Mann-Whitney $U$ test was used to compare the difference in age and other continuous variables between TM and NM. Chi-square test was performed to analyze the differences based on factors, such as gender and clinical stages. Statistical analysis was performed on SPSS version 22.0 (IBM, Armonk, NY, USA).

The robustness of features against delineation differences versus the accurate VOIs was quantified using the intra-class correlation coefficient (ICC). Features with ICC $\geq 0.9$ were considered excellent robust. The performance of diverse VOI-derived radiomics models were assessed by AUC, and the differences were compared by the method of DeLong et al. [34] using the MedCalc version 15.2.2 (MedCalc Software bvba, Ostend, Belgium). Note that a two-tailed $p$ value less than 0.05 indicated statistical significance in this work.

\section{Results}

For the metastasis differentiation in NPC before radiotherapy, no significant differences were observed between NM and TM groups except in histologic grade $(p<0.05$; Table 1$)$. In the prediction of SLN metastasis in breast cancer, NM and TM groups had no significant differences in all characteristics $(p>0.05$; Table 1$)$. Inter- observer differences are summarized in Fig. 3. Colors in the heatmap indicated that margin differences of ROIs from two radiologists were concentrated between 0 and 8 pixels for all datasets.

\section{Feature robustness analysis}

ICCs for features against all VOI variations were distributed in a wide range for all scans (Fig. 4a and b). ICC values in Smoothing which represented the smallest differences were most concentrated with the smallest effect on feature values, except for T2-FS images, for which Dilation had more concentrated distribution with the narrowest ICC range of 0.134-0.999. Dilation7 which changed the most in VOIs, revealed the largest ICC range with the great effect on feature values. The features extracted from breast cancer data were more sensitive to VOI variations compared with NPC, showing fewer robust features as a whole (Fig. 4c). Smoothing resulted in the maximum number of robust features, whereas Dilation7 worked the other way around.

\section{Feature selection analysis}

As a matter of convenience, the top-predictive features selected from diverse VOIs were re-indexed according to feature type. Each symbol in Fig. 5 represents one type of feature, and features in area filled with gray represent the same top-predictive features as accurate VOIs. The features selected under diverse VOIs showed considerable differences (Fig. 5), which indicated great effects of delineation differences on feature selection. Under any variation in the two tasks, not more than 15\% top-predictive features were identical to the accurate VOIs, particularly for CET1-w images. This result was the case for no common features. Analogously, there was a large difference in features contributing the best radiomics models (see solidfilled symbols in Fig. 5).

\section{Prediction performance analysis}

As seen in Table 3, the differences of AUCs in Smoothing and Dilation models were not statistically significant with the Baseline model except for T2-FS images in SLN group, the average AUCs of which were much higher (Smoothing: 0.845 vs. $0.725, p=0.001$; Dilation: 0.800 vs. 


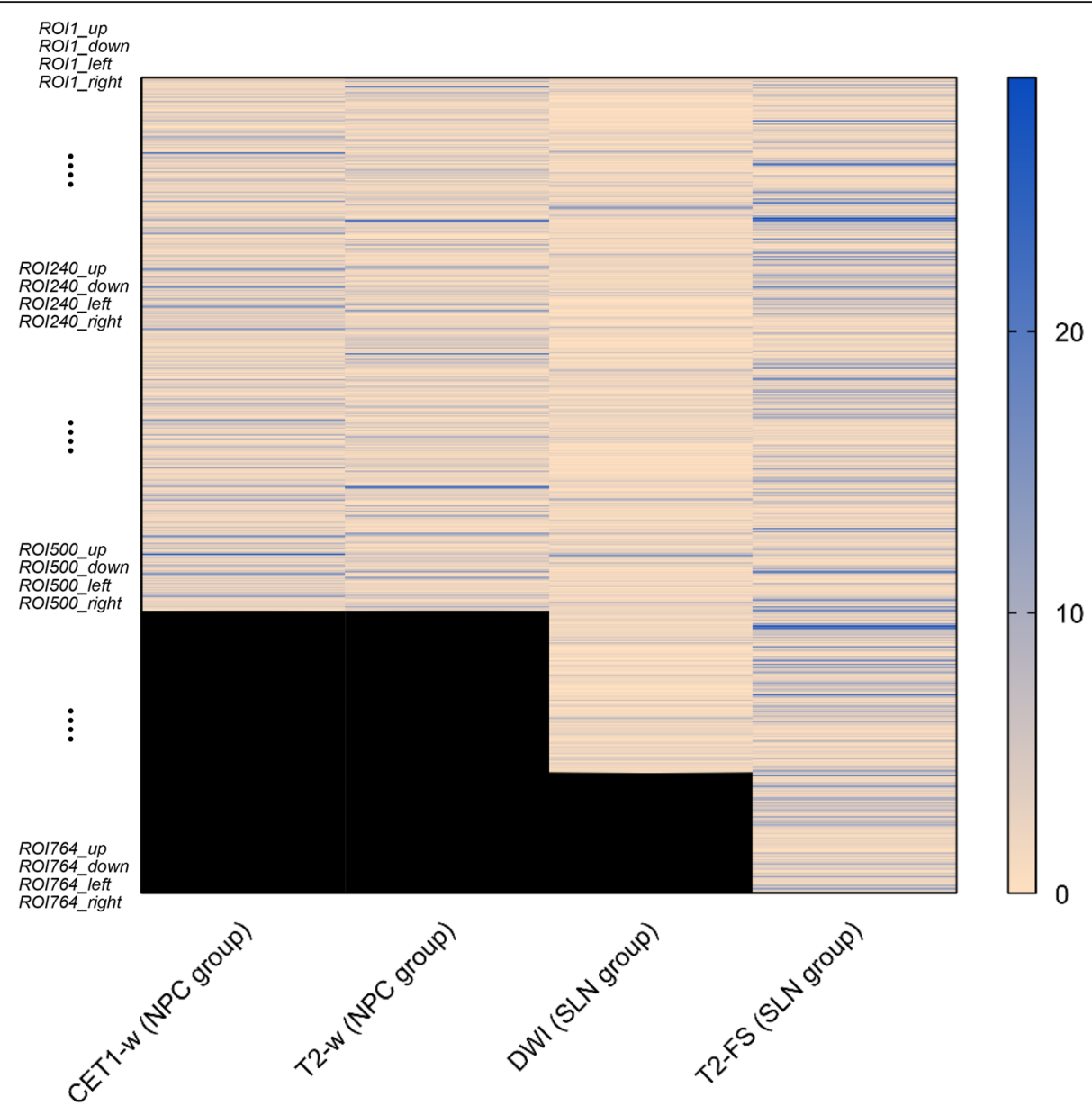

Fig. 3 Heatmap for the inter-observer analysis of the segmentation. 30 cases with segmentation by two radiologists were randomly selected from each dataset. For each selected region of interest (ROI), the smallest rectangle that best fits the tumor region was used to calculate margin distance of two kinds of manual segmentation in four directions (up, down, left, and right), resulting in multiple calculated values (number of selected ROIs $\times 4$ ) for analysis together. Colors in the heatmap indicated that margin differences of ROls were concentrated between 0 and 8 pixels for all datasets

$0.725, p=0.042)$. Erosion, which performed similarly to Smoothing and Dilation model in NPC group, performed the worst in SLN group, especially for DWI with significant differences in comparison with Baseline model $(p<0.001)$. Besides, Dilation5 and Dilation7 model contributed remarkable predictive AUCs in SLN group but the opposite in NPC group. The prediction performance of the training cohorts is shown in Additional file 1: Table S2.

Model performance across testing data from diverse VOls On the basis of feature parameters obtained from the radiomics models, we assessed stability by validating the model using data from diverse VOIs, as shown in Fig. 6. The predictive AUCs using CET1-w images, trained by data from the Dilation7 model and tested by data from other VOIs, were all above 0.7 and seemed relatively stable. Poor prediction results were still represented by Dilation5 and Dilation7 models using T2-w images. The prediction results changed in relatively large ranges across different VOI-operated validation data in SLN group. The model with training and validation data undergoing the same delineation outperformed other models in most cases.

\section{Discussion}

In this work, we investigated the influence of tumor delineation on radiomics analysis in detail within two disease groups. The tolerance of the delineation differences was explored to provide references for tumor delineation in future radiomics studies. Application of various operations to VOIs corresponded to diverse delineations in clinical practice. The results illustrated that delineation differences of VOIs had an effect on the radiomics feature values, feature selection, and prediction performance which depended on specific disease as well as MRI sequences. 
a

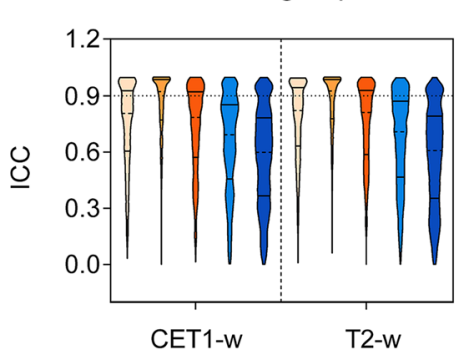

b

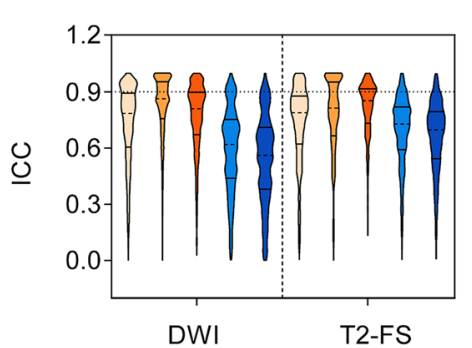

$\square$ Erosion

$\square$ Smoothing

$\square$ Dilation

$\square$ Dilation5

Dilation7

C

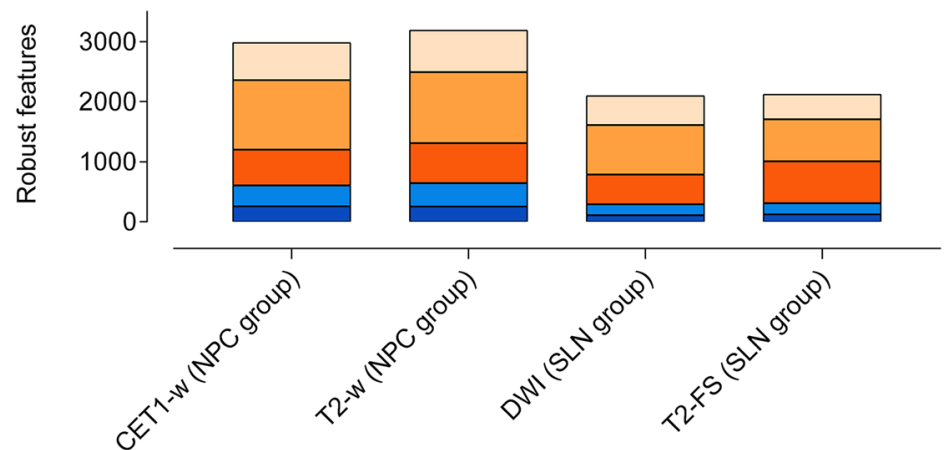

Fig. 4 Comparison of the effects of VOI differences on radiomics feature values. ICC violin plots of the radiomics features derived from diverse VOls for (a) NPC group and (b) SLN group. The dashed lines represent the median, and the solid lines represent interquartile range. (c) Number of robust features identified from diverse VOIs. Features with ICC $\geq 0.9$ were considered robust

a

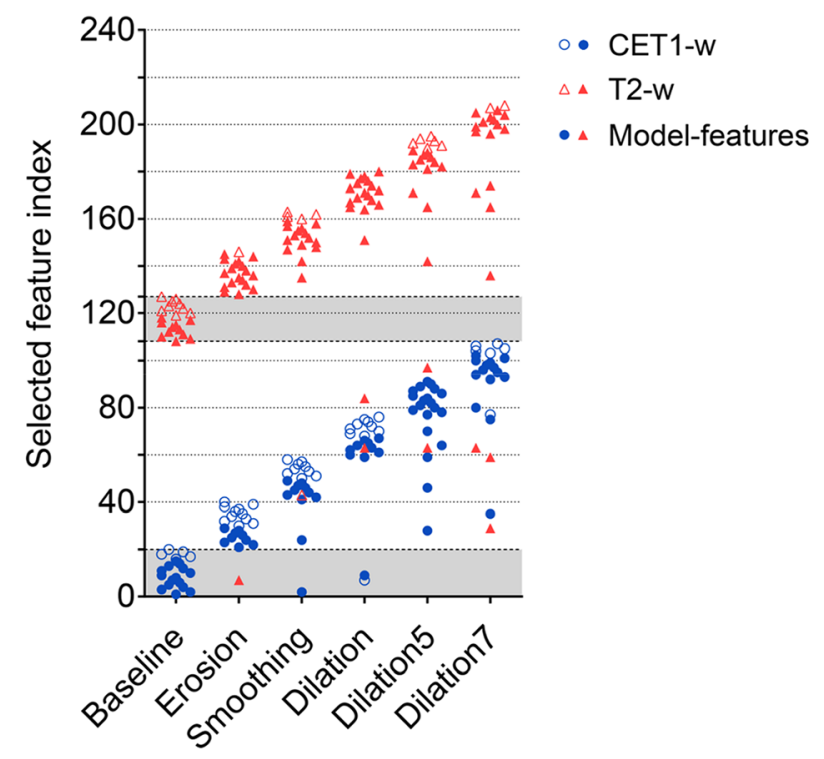

b

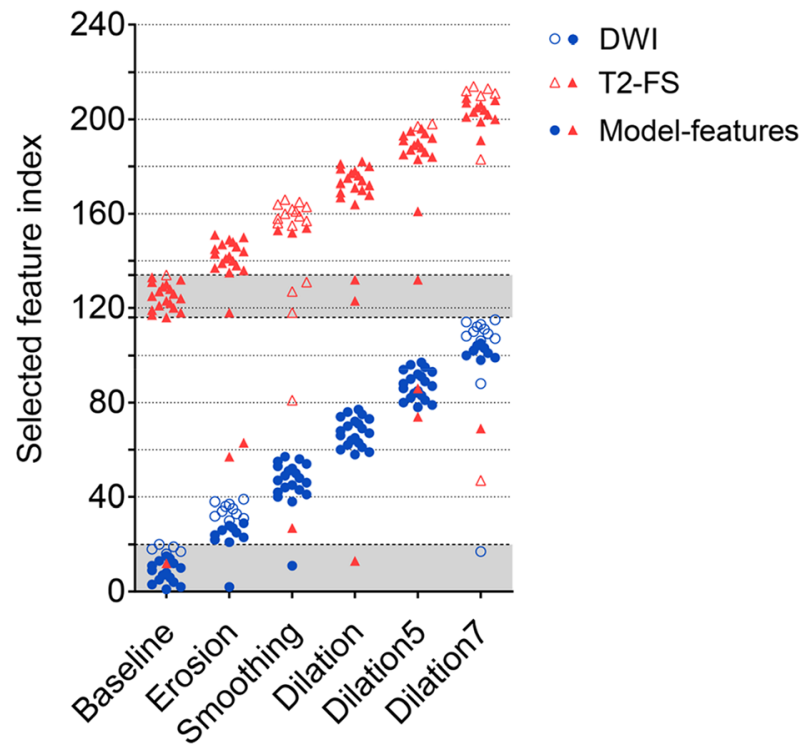

Fig. 5 Top-predictive features across feature selection from diverse VOls: (a) NPC group and (b) SLN group. Each symbol represents one type of feature, and the area filled with gray represents the range of feature types selected using accurate VOls. Twenty top-predictive features are identified for each scan, and features in area filled with gray represent the same top-predictive features as accurate VOls. Note the solid-filled symbols represent features that contribute to the best radiomics models 
Table 3 Prediction results of radiomics models from diverse VOls on the independent validation cohorts

\begin{tabular}{|c|c|c|c|c|c|c|c|c|c|}
\hline Dataset & Image & Radiomics model & Feature number & $A \cup C$ & $95 \% \mathrm{Cl}$ & $p$-Value & SEN & SPE & ACCU \\
\hline \multirow[t]{12}{*}{ NPC group } & \multirow[t]{6}{*}{ CET1-w } & Baseline & 15 & 0.778 & $0.720-0.830$ & - & $77.4 \%$ & $75.0 \%$ & $72.7 \%$ \\
\hline & & Erosion & 9 & 0.806 & $0.750-0.855$ & 0.403 & $77.4 \%$ & $85.7 \%$ & $76.9 \%$ \\
\hline & & Smoothing & 11 & 0.803 & $0.746-0.852$ & 0.412 & $80.6 \%$ & $75.0 \%$ & $74.1 \%$ \\
\hline & & Dilation & 10 & 0.810 & $0.754-0.858$ & 0.268 & $80.6 \%$ & $71.4 \%$ & $72.0 \%$ \\
\hline & & Dilation5 & 20 & 0.751 & $0.691-0.805$ & 0.437 & $87.1 \%$ & $53.6 \%$ & $68.1 \%$ \\
\hline & & Dilation7 & 14 & 0.738 & $0.678-0.793$ & 0.220 & $77.4 \%$ & $67.9 \%$ & $68.3 \%$ \\
\hline & \multirow[t]{6}{*}{$\mathrm{T} 2-\mathrm{w}$} & Baseline & 11 & 0.748 & $0.688-0.802$ & - & $74.2 \%$ & $71.4 \%$ & $70.8 \%$ \\
\hline & & Erosion & 19 & 0.710 & $0.647-0.767$ & 0.359 & $71.0 \%$ & $71.4 \%$ & $68.3 \%$ \\
\hline & & Smoothing & 15 & 0.702 & $0.639-0.759$ & 0.279 & $74.2 \%$ & $60.7 \%$ & $66.1 \%$ \\
\hline & & Dilation & 20 & 0.718 & $0.656-0.774$ & 0.447 & $77.4 \%$ & $60.7 \%$ & $65.8 \%$ \\
\hline & & Dilation5 & 14 & 0.596 & $0.530-0.659$ & $0.003^{*}$ & $54.8 \%$ & $53.6 \%$ & $53.4 \%$ \\
\hline & & Dilation7 & 18 & 0.588 & $0.522-0.651$ & $0.002^{*}$ & $61.3 \%$ & $64.3 \%$ & $57.3 \%$ \\
\hline \multirow[t]{12}{*}{ SLN group } & \multirow[t]{6}{*}{ DWI } & Baseline & 15 & 0.734 & $0.666-0.794$ & - & $77.8 \%$ & $67.7 \%$ & $69.0 \%$ \\
\hline & & Erosion & 10 & 0.536 & $0.500-0.607$ & $<0.001^{*}$ & $55.6 \%$ & $58.1 \%$ & $54.7 \%$ \\
\hline & & Smoothing & 20 & 0.711 & $0.642-0.773$ & 0.617 & $72.2 \%$ & $67.7 \%$ & $63.9 \%$ \\
\hline & & Dilation & 20 & 0.737 & $0.670-0.798$ & 0.934 & $77.8 \%$ & $64.5 \%$ & $66.7 \%$ \\
\hline & & Dilation5 & 20 & 0.744 & $0.677-0.803$ & 0.934 & $66.7 \%$ & $80.6 \%$ & $68.2 \%$ \\
\hline & & Dilation7 & 8 & 0.789 & $0.725-0.843$ & 0.269 & $83.3 \%$ & $74.2 \%$ & $72.9 \%$ \\
\hline & \multirow[t]{6}{*}{ T2-FS } & Baseline & 19 & 0.725 & $0.657-0.786$ & - & $61.1 \%$ & $74.2 \%$ & $66.5 \%$ \\
\hline & & Erosion & 20 & 0.696 & $0.627-0.760$ & 0.319 & $55.6 \%$ & $77.4 \%$ & $61.0 \%$ \\
\hline & & Smoothing & 4 & 0.845 & $0.787-0.893$ & $0.001^{*}$ & $72.2 \%$ & $74.2 \%$ & $70.6 \%$ \\
\hline & & Dilation & 20 & 0.800 & $0.737-0.854$ & $0.042^{*}$ & $66.7 \%$ & $74.2 \%$ & $70.8 \%$ \\
\hline & & Dilation5 & 18 & 0.868 & $0.813-0.912$ & $<0.001^{*}$ & $77.8 \%$ & $87.1 \%$ & $76.5 \%$ \\
\hline & & Dilation7 & 13 & 0.802 & $0.739-0.855$ & $0.028^{*}$ & $83.3 \%$ & $67.7 \%$ & $72.9 \%$ \\
\hline
\end{tabular}

Baseline no processing with the accurate VOls, Erosion erosion operation, Smoothing smoothing operation, Dilation dilation with structural element radius size of 3 , Dilation5 dilation with structural element radius size of 5, Dilation7 dilation with structural element radius size of 7, AUC area under receiver operating characteristic curve, $C I$ confidence interval, SEN sensitivity, SPE specificity, ACCU accuracy, ${ }^{*} p<0.05$ with DeLong test representing statistically significant difference in VOI-operated model and Baseline model

The experiment results provided strong evidence that the larger the VOIs changed, the greater the influence on the feature values (Fig. 4). According to the number of robust features, different diseases had discrepant sensitivity to VOI variations, consistent with a previous discovery [18]. This result could be explained from the fact that the tumors in breast cancer are larger with illdefined margins, which cause great changes on the feature values across larger variation. A comparison of toppredictive features showed that even slight smoothing on VOIs could lead to large differences in feature selection. This agrees with the discovery in [19], only one texture feature appeared on both contour-focused segmentation and the one with shrinkage of $2 \mathrm{~mm}$. Probably because the variations exactly weaken the correlation with the class of certain features by changing the feature values, which resulted in a new order of top-predictive features. A feature possessing good distinguishing characteristics does not stand out under all conditions, and thus depended on the specific analysis task.
The study also demonstrated that delineation differences of VOIs affected prediction performance of radiomics models. Stable and prominent performance from VOIs across Smoothing and Dilation indicated the tolerance of corresponding differences for radiomics models and corroborated the feasibility that the radiologists smoothly outline the lesions or slightly larger of 3 pixels width around the tumor. Note that it is not that bigger is better for VOIs. The worse performance from VOIs across Dilation5 and Dilation 7 in NPC group (Fig. 2) could be explained by the dilated area that contained more areas of the nasal cavity which exhibits low-signal intensity. This increased the effect of certain features, tending to confusion classification and facilitating feature sets with poor differentiation property. However, in breast images, more soft tissues containing complex textures were associated to capture heterogeneity for predicting SLN metastasis, indicating that the peritumoral regions had a positive influence to a certain extent. This finding is consistent with past researches 


\section{a}

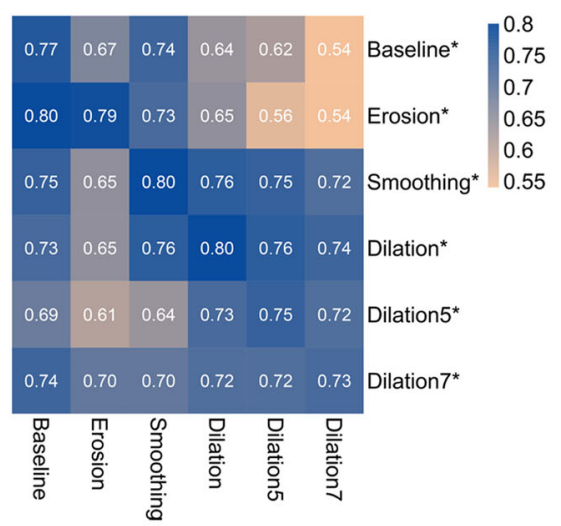

C

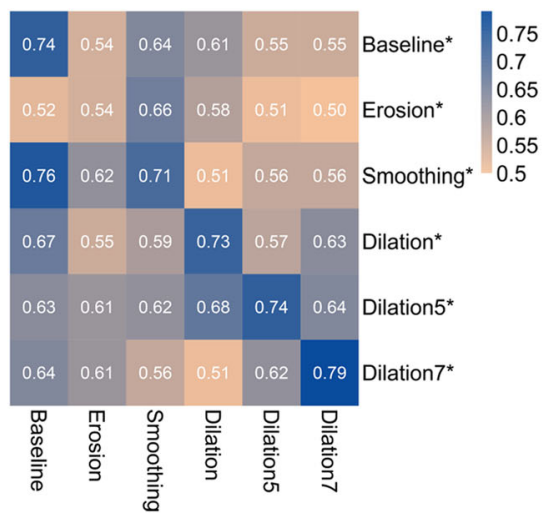

b

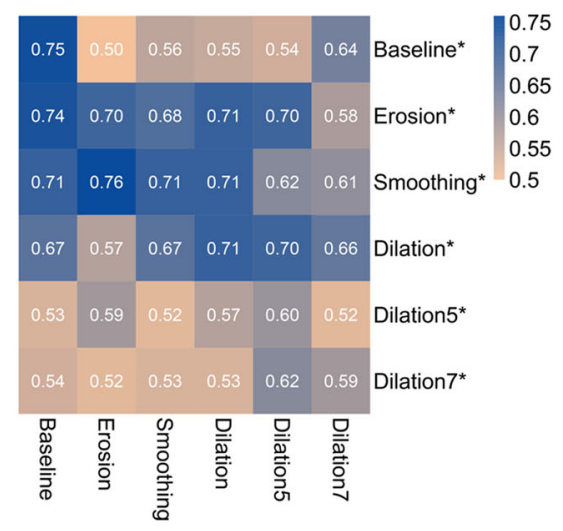

d

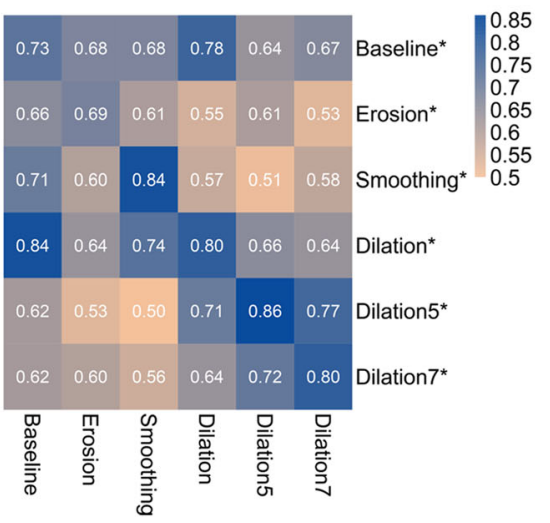

Fig. 6 Prediction performance of the radiomics models across different VOls-operated validation data. NPC group: (a) CET1-w images and (b) T2w images; SLN group: (c) DWI and (d) T2-FS images. Note that the abbreviation marked with an asterisk represents a training model. For example, Erosion* is the training model built with data and parameters obtained from VOls with erosion. The results in the first row represent the prediction performance of the Baseline model tested by different VOls-operated data

$[35,36]$. Braman et al. showed that the textural analysis of peritumoral regions contributed to the prediction of pathological complete response in neoadjuvant chemotherapy on pretreating breast cancer DCE-MRI [36]. This explanation also holds true for the worse performance from VOIs across erosion.

The radiomics models with good predictive properties might not necessarily perform well on the validation data from VOIs of diverse delineations, which implied that the VOIs of training and validation data should be outlined on the basis of the same criterion. In this regards, a unified standard should be referred in the delineation of VOIs, e.g., slight larger delineation with 3 pixels width around the tumors or lesions for all images. We suppose this assists in more accurate analysis, as the same proposal by Welch et al. [37]. In particular, the Dilation7 model distinctly reflected stable performance against all the variations using CET1-w images. The modeling features are shown in Additional file 1: Table S3. Beyond our expectation, no features showed high robustness, whether in one or all variations. We can infer that features which are not robust to the differences in VOIs may not result in poor prediction performance, which is similar with the observation of past researches $[38,39]$. The results also confirmed the insufficiency of simply analyzing the effects of differences on features robustness. In fact, whether the final performance of the radiomics models exist substantial differences is the most important issue, as emphasized in [40].

The present work also has several generalizability issues and limitations. First, while the number of patient population was small, an independent validation cohort was divided for radiomics model evaluation devoid of information leakage between feature selection/training phases. We believe this makes the results reliable and generalizable. It is in demand of more patient data for stronger verification in future research. Second, we used simple morphological operations to change tumor margin. Other contour randomization processing methods that provide stochastic components in the delineation of VOIs are lacking. For the purpose of determining the feasibility of alternative delineation of VOIs, relative 
changing of the tumor-focused delineation is easier to implement from a medical point of view. Third, regarding the differences in image resolutions between MR images, we changed the size of VOIs at pixel level to better adapt to the delineation of different scenarios. As radiologists delineate the VOIs in term of the original images, which does not involve image resampling and additional preprocessing. Fourth, the effects of the diverse delineations were analyzed and synthesized in spite of differences in tumor location and imaging manifestations within two disease groups. More types of diseases should be further assessed to provide more comprehensive references. Additionally, we only assessed the effects of diverse delineations of VOIs using MRI. The effect on the radiomics analysis for other modalities, such as PET/ $\mathrm{CT}$, is still unclear.

\section{Conclusions}

The differences in delineation of VOIs could lead to considerable differences in feature value and feature selection. The influence on prediction depended on specific disease as well as MRI sequences, among which smooth or slight larger delineation with 3 pixels width around the tumors or lesions were feasible. In addition, predefining a unified standard is suggested in the delineation to promote reliable analysis. Despite several limitations, we believe these findings are of great significance as a reference for tumor delineation in future radiomics analysis.

\section{Supplementary information}

Supplementary information accompanies this paper at https://doi.org/10. 1186/s40644-019-0276-7

Additional file 1. Note $\mathbf{S 1}$ : the inclusion criteria and flow chart of the patient data. Note S2: radiomics features. Note S3: 0.632+ bootstrap feature selection. Figure S1. The inclusion criteria and flow chart of nasopharyngeal carcinoma data. Figure S2. The inclusion criteria and flow chart of breast cancer data. Table S1. Radiomics features type and number. Table S2. Prediction results of radiomics models from diverse VOls on the training cohorts of two disease groups. Table S3. Radiomics features for the Dilation7 (dilation with structural element radius size of 7) model as well as the ICCs for metastasis estimation in nasopharyngeal carcinoma

\footnotetext{
Abbreviations

AUC: Area under the ROC curve; CET1-w: Contrast-enhanced T1-weighted; CT: Computed tomography; DWl: Diffusion-weighted image; GLCM: Greylevel co-occurrence matrix; GLRLM : Grey-level run length matrix; GLSZM: Grey-level size zone matrix; ICC: Intra-class correlation coefficient: MRI: Magnetic resonance imaging; NGTDM: Neighbourhood grey-tone difference matrix; NM : Non-metastasizing; NPC : Nasopharyngeal carcinoma; PET: Positron emission tomography; ROC: Receiver operating characteristic; ROI: Region of interest; SLN: Sentinel lymph node; T2-FS : T2-weighted fat suppression; T2-w: T2-weighted; TM: Metastasizing; VOI: Volume of interest
}

\section{Authors' contributions}

WY, LL, QF and XZ contributed to the design of the study and the machine learning method development. BZ, LZ and SZ collected and analyzed the $M R I$ data. $X Z$, LZ and BZ drafted the manuscript. HD and $L Z$ helped perform statistical analysis. All authors participated in revising the manuscript and approved the final manuscript.

\section{Funding}

This study was supported by the National Natural Science Foundation of China (No. 81771916, No. 81871323, No. 81801665) and Guangdong

Provincial Key Laboratory of Medical Imaging Processing (No. 2014B03031042).

\section{Availability of data and materials}

The data that support the findings of this study are available from corresponding author upon reasonable request.

Ethics approval and consent to participate

This retrospective study was approved by the local institutional review board with a waiver of the written informed consent from patients.

Consent for publication

Not applicable.

\section{Competing interests}

The authors declare that they have no competing interests.

\section{Author details}

${ }^{1}$ School of Biomedical Engineering, Southern Medical University, No.1023 Shatai Road, Baiyun District, Guangzhou 510515, Guangdong, China.

${ }^{2}$ Department of Radiology, The First Affiliated Hospital, Jinan University, No. 613 Huangpu West Road, Tianhe District, Guangzhou 510627, Guangdong, China. ${ }^{3}$ Department of Radiology, Guangdong General Hospital/Guangdong Academy of Medical Sciences, No. 106 Zhongshan Er Road, Yuexiu District, Guangzhou 510080, Guangdong, China.

Received: 8 October 2019 Accepted: 6 December 2019

Published online: 21 December 2019

References

1. Sun $H$, Chen $Y$, Huang $Q$, Lui S, Huang $X$, Shi $Y$, et al. Psychoradiologic utility of MR imaging for diagnosis of attention deficit hyperactivity disorder: a Radiomics analysis. Radiol 2017;287(2):620-630. https://doi.org/10.1148/ radiol.2017170226.

2. Port JD. Diagnosis of attention deficit hyperactivity disorder by using MR imaging and Radiomics: a potential tool for clinicians. Radiology. 2018; 287(2):631-632. https://doi.org/10.1148/radiol.2018172804.

3. Coroller TP, Grossmann P, Hou Y, Rios VE, Leijenaar RT, Hermann G, et al. CT-based radiomic signature predicts distant metastasis in lung adenocarcinoma. Radiother Oncol 2015;114(3):345-350. https://doi.org/10.1016/j.radonc.2015.02.015.

4. Ingrisch M, Schneider MJ, Nörenberg D, Negrao dFG, Maier-Hein K, Suchorska B, et al. Radiomic analysis reveals prognostic information in T1weighted baseline magnetic resonance imaging in patients with Glioblastoma. Investig Radiol 2017;52(6):360-366. https://doi.org/10.1097/RLI. 0000000000000349.

5. Teruel JR, Heldahl MG, Goa PE, Pickles M, Lundgren S, Bathen TF, et al. Dynamic contrast-enhanced MRI texture analysis for pretreatment prediction of clinical and pathological response to neoadjuvant chemotherapy in patients with locally advanced breast cancer. NMR Biomed 2014;27(8):887-896. https://doi.org/10.1002/nbm.3132.

6. Shiradkar R, Podder TK, Algohary A, Viswanath S, Ellis RJ, Madabhushi A. Radiomics based targeted radiotherapy planning (rad-TRaP): a computational framework for prostate cancer treatment planning with MRI. Radiat Oncol 2016;11(1):148. https://doi.org/10.1186/s13014-016-0718-3.

7. Emir UE, Larkin SJ, De PN, Voets N, Plaha P, Stacey R, et al. Noninvasive quantification of 2-Hydroxyglutarate in human Gliomas with IDH1 and IDH2 mutations. Cancer Res 2015:76(1):43-49. https://doi.org/10.1158/0008-5472.CAN-15-0934.

8. Huang Y, Liu Z, He L, Chen X, Pan D, Ma Z, et al. Radiomics signature: a potential biomarker for the prediction of disease-free survival in early-stage 
(I or II) non-small cell lung Cancer. Radiology. 2016;281(3):947-957. https://doi.org/10.1148/radiol.2016152234.

9. Lambin P, Rios-Velazquez E, Leijenaar R, Carvalho S, Stiphout RGPMV, Granton P, et al. Radiomics: extracting more information from medical images using advanced feature analysis. Eur J Cancer 2012;48(4):441-446. https://doi.org/10.1016/.jejca.2011.11.036.

10. Baumann M, Krause M, Overgaard J, Debus J, Bentzen SM, Daartz J, et al. Radiation oncology in the era of precision medicine. Nat Rev Cancer 2016; 16(4):234-249. https://doi.org/10.1038/nrc.2016.18.

11. Gillies RJ, Kinahan PE, Hricak H. Radiomics: images are more than pictures, They Are Data Radiology 2016;278(2):563-577. https://doi.org/10.1148/radiol. 2015151169.

12. Vallières M, Freeman CR, Skamene SR, El NI. A radiomics model from joint FDG-PET and MRI texture features for the prediction of lung metastases in soft-tissue sarcomas of the extremities. Phys Med Biol 2015;60(14):54715496. https://doi.org/10.1088/0031-9155/60/14/5471.

13. Lu L, Lv W, Jiang J, Ma J, Feng Q, Rahmim A, et al. Robustness of Radiomic features in [(11) C] choline and [(18) F] FDG PET/CT imaging of nasopharyngeal carcinoma: impact of segmentation and discretization. Mol Imaging Biol 2016;18(6):935-945. https://doi.org/10.1007/s11307-016-0973-6.

14. Bagher-Ebadian H, Siddiqui F, Liu C, Movsas B, Chetty IJJMP. On the impact of smoothing and noise on robustness of $C T$ and $C B C T$ radiomics features for patients with head and neck cancers. Int J Radiat Oncol Biol Phys 2017; 99(2):S93. https://doi.org/10.1016/j.jirobp.2017.06.225.

15. Shiri I, Rahmim A, Ghaffarian P, Geramifar P, Abdollahi H, Bitarafan-Rajabi AJER. The impact of image reconstruction settings on 18F-FDG PET radiomic features: multi-scanner phantom and patient studies. Eur Radiol 2017;27(11):4498-4509. https://doi.org/10.1007/s00330-017-4859-z.

16. Leunens G, Menten J, Weltens C, Verstraete J, Van dSE. Quality assessment of medical decision making in radiation oncology: variability in target volume delineation for brain tumours. Radiother Oncol 1993;29(2):169-175. https://doi.org/10.1016/0167-8140(93)90243-2.

17. Belli ML, Mori M, Broggi S, Cattaneo GM, Bettinardi V, Dell'Oca I, et al, Quantifying the robustness of [18F]FDG-PET/CT radiomic features with respect to tumor delineation in head and neck and pancreatic cancer patients. Phys Medica 2018;49:105-111. https://doi.org/10.1016/j.ejmp.2018.05.013.

18. Pavic M, Bogowicz M, Würms $X$, Glatz $S$, Finazzi T, Riesterer $\mathrm{O}$, et al. Influence of inter-observer delineation variability on radiomics stability in different tumor sites. Acta Oncol 2018:57(8): 1070-1074. https://doi.org/10. 1080/0284186X.2018.1445283.

19. Kocak B, Ates E, Durmaz ES, Ulusan MB, Kilickesmez O. Influence of segmentation margin on machine learning-based high-dimensional quantitative $C T$ texture analysis: a reproducibility study on renal clear cell carcinomas. Eur Radiol 2019;29(9): 4765-4755. https://doi.org/10.1007/ s00330-019-6003-8.

20. Rexilius J, Hahn HK, Schlüter M, Bourquain H, Peitgen HO. Evaluation of accuracy in MS lesion volumetry using realistic lesion phantoms. Acad Radiol 2005;12(1):17-24. https://doi.org/10.1016/j.acra.2004.10.059.

21. Lee AW, Poon YF, Foo W, Law SC, Cheung FK, Chan DK, et al. Retrospective analysis of 5037 patients with nasopharyngeal carcinoma treated during 1976-1985: overall survival and patterns of failure. Int J Radiat Oncol Biol Phys 1992;23(2):261-270. https://doi.org/10.1016/0360-3016(92)90740-9.

22. Geara FB, Sanguineti G, Tucker SL, Garden AS, Ang KK, Morrison WH, et al. Carcinoma of the nasopharynx treated by radiotherapy alone: determinants of distant metastasis and survival. Radiother Oncol 1997;43(1):53-61. https://doi.org/10.1016/S0167-8140(97)01914-2.

23. Tang L, Li L, Mao Y, Liu L, Liang S, Chen Y, et al. Retropharyngeal lymph node metastasis in nasopharyngeal carcinoma detected by magnetic resonance imaging : prognostic value and staging categories. Cancer. 2008; 113(2):347-354. https://doi.org/10.1002/cncr.23555.

24. Dong Y, Feng Q, Yang W, Lu Z, Deng C, Zhang L, et al. Preoperative prediction of sentinel lymph node metastasis in breast cancer based on radiomics of T2-weighted fat-suppression and diffusion-weighted MRI. Eur Radiol 2018;28(2):582-591. https://doi.org/10.1007/s00330-017-5005-7.

25. Tustison NJ, Avants BB, Cook PA, Zheng Y, Egan A, Yushkevich PA, et al. N4ITK: improved N3 Bias correction. IEEE Trans Med Imaging 2010;29(6): 1310-1320. https://doi.org/10.1109/TMI.2010.2046908

26. Nyul $L G$, Udupa JK, Zhang $X$. New variants of a method of MRI scale standardization. IEEE Trans Med Imaging 2000;19(2):143-150. https://doi.org/10.1109/42.836373.
27. Yushkevich PA, Piven J, Hazlett HC, Smith RG, Ho S, Gee JC, et al. Userguided $3 \mathrm{D}$ active contour segmentation of anatomical structures: significantly improved efficiency and reliability. Neuroimage. 2006;31(3): 1116-1128. https://doi.org/https://doi.org/10.1016/j.neuroimage.2006.01.015.

28. Galloway MM. Texture analysis using gray level run lengths. Computer Graphics \& Image Processing 1975;4(2):172-179. https://doi.org/10.1016/ S0146-664X(75)80008-6.

29. Amadasun M, King R. Textural features corresponding to textural properties, IEEE Transactions on Systems, Man and Cybernetics 1989;19(5):1264-1274. https://doi.org/10.1109/21.44046.

30. Thibault G, Fertil B, Navarro C, Pereira S, Cau P, Levy N, et al. Shape and texture indexes application to cell nuclei classification. Int J Pattern Recogn 2013;27(1):1357002. https://doi.org/10.1142/S0218001413570024.

31. Peng $\mathrm{H}$, Long $\mathrm{F}$, Ding $\mathrm{CHQ}$. Feature selection based on mutual information criteria of max-dependency, max-relevance, and min-redundancy. IEEE T Pattern Anal 2005;27(8):1226-1238. https://doi.org/10.1109/TPAMI.2005.159.

32. Sahiner B, Chan HP, Hadjiiski L. Classifier performance prediction for computer-aided diagnosis using a limited dataset. Med Phys 2008;35(4): 1559-1570. https://doi.org/10.1118/1.2868757.

33. Breiman LJML. Random forests. Mach Learn 2001;45(1):5-32. https://doi.org/ 10.1023/A:1010933404324.

34. Delong ER, Delong DM, Clarkepearson DL. Comparing the areas under two or more correlated receiver operating characteristic curves: a nonparametric approach. Biometrics. 1988;44(3):837-45 https://doi.org/10.2307/2531595.

35. Prasanna P, Patel J, Partovi S, Madabhushi A, Tiwari P. Radiomic features from the peritumoral brain parenchyma on treatment-naïve multiparametric MR imaging predict long versus short-term survival in glioblastoma multiforme: preliminary findings. Eur Radiol 2017;27(10):41884197. https://doi.org/10.1007/s00330-016-4637-3.

36. Braman NM, Etesami M, Prasanna P, Dubchuk C, Gilmore H, Tiwari P, et al. Intratumoral and peritumoral radiomics for the pretreatment prediction of pathological complete response to neoadjuvant chemotherapy based on breast DCE-MRI. Breast Cancer Res 2017;19(1):57. https://doi.org/10.1186/ s13058-017-0846-1.

37. Welch ML, Mcintosh C, Haibekains B, Milosevic M, Wee L, Dekker A, et al. Vulnerabilities of radiomic signature development: the need for safeguards. Radiother Oncol 2019;130:2-9. https://doi.org/10.1016/j.radonc.2018.10.027.

38. Hatt, Mathieu, Tixier, Florent, Visvikis, Dimitris, et al. robustness of intratumour F-18-FDG PET uptake heterogeneity;quantification for therapy response prediction in oesophageal carcinoma. Eur J Nucl Med Mol Imaging 2013;40(11):1662-1671. https://doi.org/10.1007/s00259-013-2486-8.

39. Lv W, Yuan $Q$, Wang $Q, M a ~ J$, Jiang J, Yang W, et al. Robustness versus disease differentiation when varying parameter settings in radiomics features: application to nasopharyngeal PET/CT. Eur Radiol 2018;28(8):32453254. https://doi.org/10.1007/s00330-018-5343-0.

40. Rios E, Parmar C, Jermoumi M, Aerts H. TU-A-12A-10: robust Radiomics feature quantification using semiautomatic volumetric segmentation. Med Phys 2014:41(6):452-452. https://doi.org/10.1118/1.4889256.

\section{Publisher's Note}

Springer Nature remains neutral with regard to jurisdictional claims in published maps and institutional affiliations.

Ready to submit your research? Choose BMC and benefit from:

- fast, convenient online submission

- thorough peer review by experienced researchers in your field

- rapid publication on acceptance

- support for research data, including large and complex data types

- gold Open Access which fosters wider collaboration and increased citations

- maximum visibility for your research: over $100 \mathrm{M}$ website views per year

At BMC, research is always in progress.

Learn more biomedcentral.com/submissions 